\title{
Stochastic control for optimal power flow in islanded microgrid
}

\author{
Thang Diep-Thanh ${ }^{1}$, Quang Nguyen-Phung ${ }^{2}$,Huy Nguyen-Duc \\ ${ }^{1,2}$ Institute for Control Engineering and Automation, Hanoi University of Science and Technology, Vietnam \\ ${ }^{3}$ School of Electrical Engineering, Hanoi University of Science and Technology, Vietnam
}

\begin{tabular}{l}
\hline \hline Article Info \\
\hline Article history: \\
Received Dec 19, 2017 \\
Revised May 23, 2018 \\
Accepted Jun 25, 2018 \\
\hline Keywords: \\
Hamilton-Jacobi-Bellman \\
equation \\
Hybrid Power System \\
Markov Process \\
Microgrid \\
Optimal Power Flow
\end{tabular}

Optimal Power Flow

\begin{abstract}
The problem of optimal power flow (OPF) in an islanded mircrogrid (MG) for hybrid power system is described. Clearly, it deals with a formulation of an analytical control model for OPF. The MG consists of wind turbine generator, photovoltaic generator, and diesel engine generator (DEG), and is in stochastic environment such as load change, wind power fluctuation, and sun irradiation power disturbance. In fact, the DEG fails and is repaired at random times so that the MG can significantly influence the power flow, and the power flow control faces the main difficulty that how to maintain the balance of power flow? The solution is that a DEG needs to be scheduled. The objective of the control problem is to find the DEG output power by minimizing the total cost of energy. Adopting the Rishel's famework and using the Bellman principle, the optimality conditions obtained satisfy the Hamilton-Jacobi-Bellman equation. Finally, numerical examples and sensitivity analyses are included to illustrate the importance and effectiveness of the proposed model.
\end{abstract}

Copyright (C) 2019 Institute of Advanced Engineering and Science. All rights reserved.

\section{Corresponding Author:}

Quang Nguyen-Phung, Institute for Control Engineering and Automation, Hanoi University of Science and Technology, 1 Dai Co Viet, Hai Ba Trung, Hanoi, Vietnam. Email: quang.nguyenphung@hust.edu.vn

\section{INTRODUCTION}

In the last decades, the earth's climate has changed much more than before, particularly the carbon dioxide level has increased faster and faster so that $\mathrm{CO}_{2}$ level surpassed $404 \mathrm{ppm}$ for the first time in recorded history as mentioned in climate.nasa.gov. In order to reduce carbon dioxide level, renewable energy source is a great solution for energy generation. From this point of view, it is expected that the renewable electricity such as wind and solar would play an important role to provide the electrical energy not only in the near future but also to islanded microgrid [1], [2]. Concerning the microgrid (MG) of which the concept was proposed and has been recently emerged, developed in many institutions to introduce a smart grid [3]. In reality, the installation of DG with small-scale power generation technologies has rapidly increased in many countries at a reduced cost and a higher efficiently. MG can operate in grid-connected, and islanded operating modes [4], [5].

The principle roles of the microgrid control structure are [4] and [5] as voltage, frequency, and angle regulation for operating modes; proper load sharing and distributed energy resources; microgrid synchronization with the main grid (utility grid); power flow control between the microgrid and the main grid, or within microgid; and optimizing the microgrid operating cost. In islanded operating mode, the local loads should be supplied by the DG units that the control is carried out as controlled voltage sources (CVS) [6]. Moreover, the MG operation can be subject to several problems such as: voltage oscillations, frequency oscillations, and power quality issues [7]. Therefore, the hierarchical control structure consists of four levels, namely, the primary, secondary, tertiary, and emergency control [4]. Literature [4] shows that the primary control is in normal operation, and droop control methods involve an inherent trade-off between power 
sharing and voltage and frequency regulation, and include fundamental control hardware. The secondary control is in off-normal operation, and compensate for the voltage and frequency deviations after the primary control achieves the power sharing. Particularly, the tertiary control is used to restore the secondary reserve, and manages the power flow between the microgid and the grid, and within the microgrid for the case of islanded mode that leads to the economic issues. Therefore, the optimal power flow is also to be called energy management that implements in a SCADA system that produces the lowest cost of energy.

To solve the problem of optimal power flow in the tertiary control level, basically we must consider the uncontrollable nature of wind, solar power as well as load change raises uncertainty for power system operation on the one part, the application and integration of DGs into the system is complex on the other part. Among these aspects are, the investigation of impact of distributed generation on the power fluctuations from penetration of wind, photovoltaic power is presented in [8], [9]. According to [10], [11] the authors have considered the hybrid power system whose the energy storage/thermal unit has a high potential for providing regulation power to meet the reverse requirements. In the context of uncertainty, the work in [12] focus on the design optimization of MG by using the stochastic method to model energy systems such that the probability of occurrence of incident solar source called Stochastic Programming method. In the literature, there are many researches that have been aimed to solve the problem optimality.

One of important works as in [13], which examines the optimal problem as the investigation of optimal power flow by adopting the interval optimization in which the wind power is defined in range of values; the interval power flow problem for DC power flow can be formulated as a non-convex and nonlinear programming. In consequence, to ensure system reliability, the forecasting uncertainty must be considered into generation scheduling, and interval power flow provides promising approach to achieve the boundary information of system statuses under uncertainties. More importantly, the contribution in [13] is a landmark for class of optimal power flow problem. For the load flow problem which is typically formulated as a set of non-linear equations as functions of bus voltages has taken some advantage. The contributions of optimal power flow are considred in [14], the control of MG in [15], and quality of power (power factor, total harmonic distortion) as in [16]. Stochastic demand has been developed and presented in [17]-[19], those authors used stochastic, fuzzy, and probability programming techniques to model the uncertainties. Although the works in [17]-[19] have been specified to load flow problem with some algorithms, the aforementioned methods are typically dependent on predefined probability distribution function or membership function of uncertainty. Furthermore, it is hard to identify accurate probability distribution functions due to data availability and stochastic nature of the uncertainty.

The research in all these directions [8]-[22] for the case of power flow was based on non-convex, and non-linear programming and the DG units have known locations and running all the time. To add to the complexity of the problem, in the real life systems, the operation of these DG units, undergoes different scenarios according to the strategies of the electricity producers and the needs of the consumers. Therefore, some uncertainties are introduced in the operation of such units and thus, stochastic modeling of systems involving DG units becomes of great interest. The sources of the uncertainties in the operation of the DG systems at a certain hour of the day include. These uncertainties affect the modeling and evaluation of the system capacity, power losses.

It's not similar to the reviewed papers above, the aim of this paper design is to focus on investigation of the power flow of hybrid wind-PV-diesel power system under uncertainties such as load change, wind power fluctuation, sun irradiation power disturbance, and the diesel engine generator fails and is repaired at random times. The objective function is to minimize the cost of electricity production. Therefore utilizes the energy balance equation in real time to formulate the optimal power flow problem as optimal control problem of a linear system by using dynamic programming. By adopting the Rishel's formalism [20], the DEG failure and repair times are characterized by Markov process and the cost function is described at terminal time. To do this it, will be expedient to derive an algorithm similar to the Bellman principle where the optimality conditions satisfy Hamilton-Jacobi-Bellman equations, and the value function is convex. In addition, one of the principle reasons for introducing feedback into an optimal control for power flow problem is to make the resulting system relatively insensitive to fluctuations that can deal with uncertainties of power system considered.

\section{HYBRID ISLAND MICROGRID}

For the simple system as shown in Figure 1, we consider the hybrid power system on the islanded AC microgrid including AC loads, photovoltaic (PV), wind turbine generator (WTG), and diesel engine generator (DEG). Also we consider a stochastic control problem for the DEG subject to failures and repairs in continuous time. 


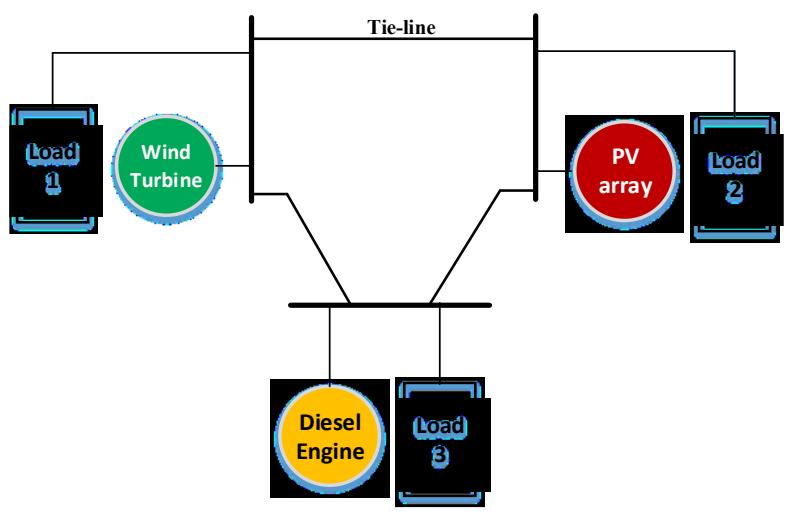

Figure 1. Simplified schematic of an islanded microgrid

As in Figure 1, the system consists of three stochastic sources such that WTG as stochastic wind power, PV as stochastic solar power, and includes the DEG that is subject to failures and repairs. On the other hand, the load demand is stochastic based on forecasting in short-term. The DGs are connected to AC bus by power electronic devices used synchronization as AC sources as DEG, WTG, and PV with invert DC voltages into AC called inverter. The DEG is used for the conversion of mechanical energy into electrical energy as controllable source.

Now, the problem is considered in finite time in order to find the DEG power generation. Such a DEG is subject to random failures and repairs and can inject the power into MG. The DEG has two possible operational states. The dynamic of the DEG (i.e., jump from one state to another) is characterized by a twostate stochastic process $\{\chi(s): s \geq 0\}$ defined as:

$$
\chi(s)=\left\{\begin{array}{l}
0 \text { if the DEG is under repair } \\
1 \text { if the DEG is fully functioning }
\end{array}\right.
$$

The process $\chi(s)$ is a controlled process, as it will depend on the DEG action when the DEG is available generation. Thus, $\{\chi(s): T \geq s \geq t\}$ is a Markov process, with value in $\{0$, and 1$\}$. Let $(\Omega, \mathrm{F}, P)$ denote the underlying probability space and let $\mathrm{F}_{t}{ }^{s}=s(c(t), t £ t £ s)$ be the sigma algebra. Let us define $F(t)$ and $G(t)$ as the functioning and repair time distributions of the DEG, respectively. Given that the DEG is operational, the probability of a failure in an interval of length $\Delta t$ is $\lambda \Delta t$. The probability that the failed DEG is repaired during such a time interval is $\mu \Delta t$. Thus, the parameters $\lambda$ and $\mu$ are the failure and repair rates for the DEG. Now, the dynamics of the DEG state are given by:

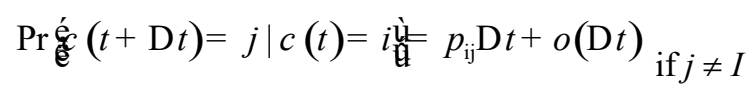

In this case, $p_{01}=\mu, p_{10}=\lambda$ are the transition probabilities.

Based on Markov properties [21], the transition probabilities are defined by

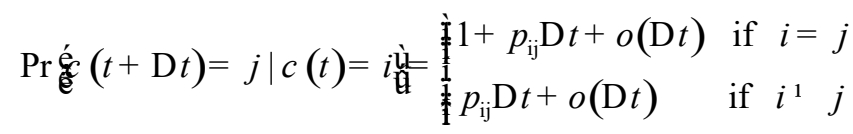

with

$$
\lim _{\mathrm{D} t \circledast 0} O(\mathrm{D} t)=0, p_{j j}=-{\stackrel{\circ}{i^{1} j}}_{i j} p_{i j} \text { for all } i, j \in \Omega .
$$

As mentioned in Section 1, the OPF problem is considered in the tertiary control level, in what following, we then describe the modeling of the considered microgrid. First of all, the total power generation of DGs satisfies the demand such as: 


$$
P_{D E G}(t)+P_{W T G}(t)+P_{P V}(t)=D(t)
$$

where $D(t)$ refers to the load demand at time $t ; P_{\mathrm{WTG}}(t)$ is the non-dispatchable (WTG) output power generation at time $t ; P_{\mathrm{PV}}(t)$ is the $\mathrm{PV}$ output power generation at time $t$; and $P_{\mathrm{DEG}}(t)$ is the DEG output power generation at time $t$. As mentioned above, the produced power by PV and WTG depends on the environmental conditions, and the demand depends on the power consumption habits. Meanwhile, the DEG is subject to random events such as failure and repair. As result, the fluctuations in loads, PV, and WTG output power are adjusted by control in the DEG output power under failure and repair events. Therefore, the expression of the power balance in Equation (5) for randomness in such DG associated with demand can be described as follows:

$$
P_{D E G}(t)=D(t)-\left(P_{W T G}(t)+P_{P V}(t)\right)
$$

The power generation of DEG must evolve the fluctuations in loads and DGs in Equation (6). In literature the optimal power flow (OPF) problem has been investigated based on linear and non-linear programming such [10], and [13]. In contrast, we will formulate the considered problem as an optimal control one in order to find out the optimal policies for operation of microgrid in the next section.

\section{MATHEMATICAL MODEL}

In this section, we consider an optimal control problem for the system described in Section 2, and assume that the DEG is subject to random events such subject to failures and repairs in continuous time called jump linear. The problem is considered in finite time (i.e., $0<T<\infty$ ) in order to find the DEG output power. In the literature, Rishel in [20] was established the necessary and sufficient condition of the stochastic control problem by the dynamic programming. In Rishel's work, the discrete jump is characterized by Markov process and the cost function described at terminal time. For more large, Rishel in [22] presents the necessary conditions by use the unnormalized conditional probabilities and expectations of partially observed jump Markov process, and the cost function defined by the integral on a finite interval. An extension of Rishel's formalism, Boukas in [23] was established the conditions for optimality for partially observed controlled with the non-homogenous Markov process. For a semi-Markov process, the transition rates depend on the duration of the system. So those transition rates can be used to model phenomena like maintenance in real time.

Clearly, to formulate a new model, by adopting Rishel's framework [20] we shall assume that the power generation in the real time, and the state equation is represented as follows:

$$
\begin{aligned}
& \frac{d X^{i}(t)}{d t}=U^{i}(t)-\left(D(t)-\left(P_{W T G}(t)+P_{P V}(t)\right)\right) \\
& \frac{d X^{i}(t)}{d t}=f^{i}(t, X(t), U(t))
\end{aligned}
$$

Constraints:

$$
\begin{aligned}
& P_{\min }^{\mathrm{W} T G}(t) £ P_{W T G}(t) £ P_{\max }^{\mathrm{WTG}}(t) \\
& P_{\min }^{P V}(t) £ P_{P V}(t) £ P_{\text {max }}^{P V}(t) \\
& P_{\min }^{D E G}(t) £ P_{D E G}(t) £ P_{\text {max }}^{D E G}(t)
\end{aligned}
$$

where $U(t)=P_{D E G}(t)$ is the control variable in equation (6) and $U(t) \hat{\mathrm{I}} \hat{\mathrm{A}}^{+}=[0,+¥)$ in $[k W], D(t)$ is load demand; $P_{W T G}(t), P_{P V}(t)$ and $P_{D E G}(t)$ are within their forecasted upper bounds $P_{\max }^{\mathrm{WTG}}(t), P_{\max }^{P V}(t), P_{\max }^{D E G}(t)$ and 
lower bounds $P_{\min }^{\mathrm{W} T G}(t), P_{\min }^{P V}(t), P_{\min }^{D E G}(t)$ (in $[k W]$ ), $X(t)$ is the difference between cumulative electric production and cumulative load demand, called surplus at time $t$ if $X(t)$ is positive and backlog if $X(t)$ is negative, shortly called energy in $[k W h]$ and $X(t) \hat{\mathrm{I}} \hat{\mathrm{A}}=(-¥,+¥), i$ is the discrete state index $i \in \Omega$ : in this case $i=0$ and $1, f^{i}(t, X, U)$ is the state function and satisfies the Lipschitz condition [24]:

$$
\left|f^{i}\left(t, X_{1}, U\right)-f^{i}\left(t, X_{2}, U\right)\right| £ K_{r}\left|X_{1}-X_{2}\right|
$$

where $K_{\rho}$ is constant.

The behavior of the state variable $X(t)$ will be specified shortly in the subsection 3.2.

Let us define cost function (cost-to-go or cost-to-arrive) which is given by

$$
J^{i}(X, t ; U)=E\left\{\int_{t}^{T} G(s, X, U) d s \mid X(t)=X, \chi(t)=i\right\}
$$

where $G(t, X(t), U(t))$ is the running cost function: $G()=.C^{+} X^{+}+C^{-} X^{-}$with $C^{+}$representing a unit surplus cost at time $t, C^{-}$the unit backlog cost at time $t, X^{+}=\max (X, 0)$, and $X^{-}=\min (0,-X)$.

Thus, the function $J^{i}(t, X, U)$ is called an overall cost of the system. To simplify things, we make the following assumptions in this paper to describe the hybrid power system:

(A.1) The total power generation satisfies the power demand in finite time considered $T$, i.e, at any time $t$ :

$$
P_{D E G}(t)+P_{W T G}(t)+P_{P V}(t)^{3} D(t)
$$

(A.2) The stochastic power values $D(t), P_{W T G}(t)$ and $P_{P V}(t)$ are forecasted values in $[\mathrm{kW}$.

\section{DEFINITION 3.1.}

(1) A control variable $Y(X, t)=\left\{U(X, t)=U(t)^{3} \quad 0\right\}$ is called an admissible control;

(2) A control $\mathrm{S}(t, X)$ is the set of admissible control $Y$ with initial vector $X(t)=X$.

Our motivation is to obtain admissible control $U(X, t) \hat{\mathrm{I}} \mathrm{S}(X, t)$ that optimizes the cost function (13). In what following, we will build the model that satisfies the contrary (3-12) and optimization of (13) by using the dynamic programming approach.

We formulate the power flow problem defined above. Under appropriate conditions, the optimal control policy is to satisfy (8-12) in order to determine the OPF $U(X, t)$ which minimize the cost function described in (13). These policies are characterized by a target production level subject to capacity constraints.

We denote $V^{i}(X, t)$ the value function, i.e.:

$$
V^{i}(X, t)=\inf _{U(.) \hat{\mathrm{S}}(t, .)} J^{i}(X, t ; U)
$$

This function will be used to establish the optimality conditions. For simplicity in the presentation of the model, we use only the sign $V^{i}(X, t)$. Based on the dynamic programming principle, the following theorem is used for the generalization of the value function in (14):

Theorem 3.1 Control problem satisfies the system of partial differential equations:

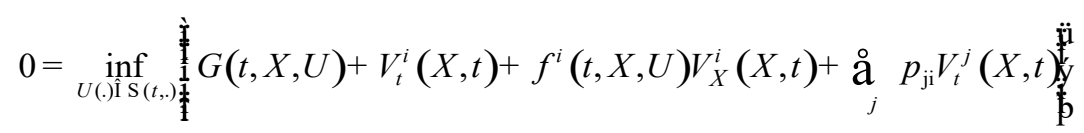


at time the initial and boundary conditions are satisfied:

$$
X(t)=X \text { for }(X, t) \hat{I} \quad Q ; V^{i}(T, X(T))=0
$$

where the terms $V_{t}^{i}(X, t)$, and $V_{X}^{i}(X, t)$ denote the gradient of value function respect to time $t$ and state variable $X$, respectively, and $Q=\left[t_{0}, T\right] \hat{\mathrm{A}}$.

The following theorem presents the necessary and sufficient conditions for which an optimal solution exists.

Theorem 3.2. Let $V^{i}(X, t) \hat{\mathrm{I}} Q$ be a solution to (15). Then for all $(X, t) \in Q$ :

(i) for every admissible control system $U(X, t)$ :

$$
V^{i}(X, t) £ J^{i}(X, t ; U)
$$

(ii) If there exists an admissible system $U^{*}(X, t)$ such that

$$
U^{*}(X, t) \hat{\mathrm{I}} \arg \min _{U(.) \mathrm{i} \mathrm{W} .)}\left\{G(t, X, U)+f^{i}(t, X, U) V_{X}^{i}(X, t)\right\}
$$

almost everywhere in t, then $V^{i}(X, t)=J^{i}\left(t, X ; U^{*}\right)$, and $U^{*}(X, t)$ is the optimal solution.

Proof. The Proofs of these theorems are adopted the Rishel's framework as in [37] and [30].

REMARKS. The system of partial differential Equation (15) is well known the Hamilton-Jacobi-Bellman (HJB) equations associated with optimal control problem under study. The optimal feedback control (15) is designed to drive the system to the optimal point (hedging point or balance point), and corresponds to the value function described by Equation (15). Then, when the value function $V^{i}(X, t)$ is available, an optimal policy can be obtained as in Equation (18). However, an analytical solution of equation (15) is so hard to find. Thus, the numerical solution of HJB Equation (18) is adopted from Kushner's method [25] to be represented in the next section.

\subsection{The feedback control}

Let $\xi(t)$ be the random parameter such as the stochastic wind power, demand, or solar radiation (called disturbance or noise dependent on context). Let $\pi(t, X(t))$ be the mapping of $U(X, t)$ such that $\pi(t, X(t)) \in \sum(X, t)$. In the Equation (7), the disturbance $\xi(t)$ may consist of $D(t), P_{\mathrm{WTG}}(t)$, and $P_{\mathrm{PV}}(t)$. Hence, the feedback control of (7) is represented in the Figure 2: 


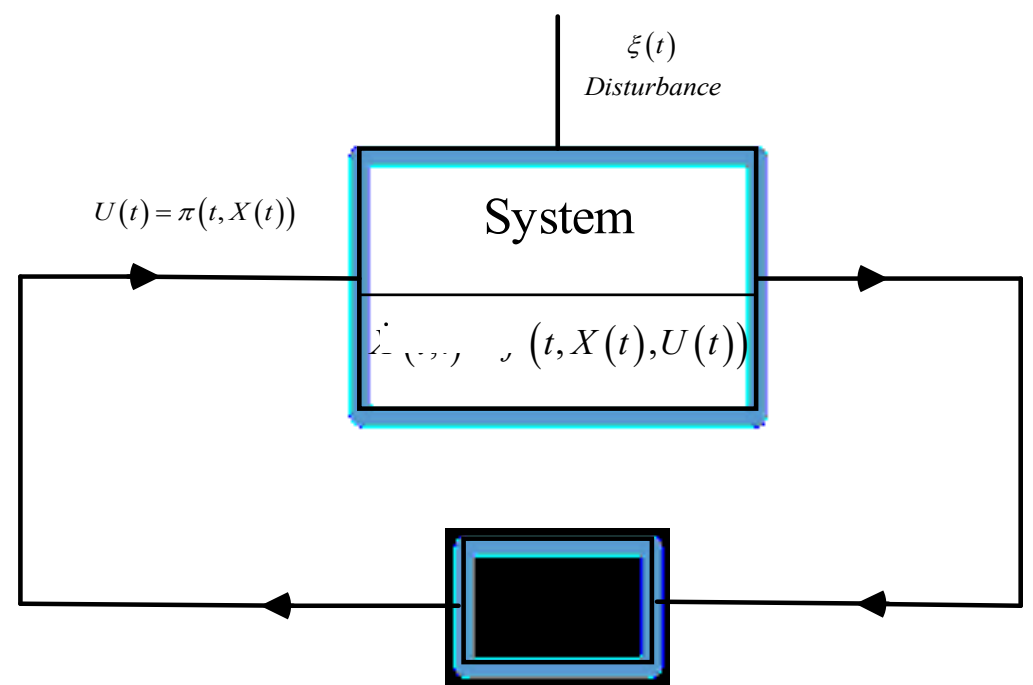

Figure 2. The closed-loop policies [26]

\subsection{Behavior of cumulative production}

By definition, the energy in equation (7) whose value is described as follows:

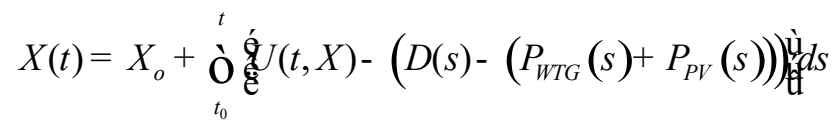

In the Figure 3, there is two kinds of regions: (1) the total power generation is less than the demand called backlog in the interval $\left(0, t_{1}\right),(2)$ it is more than the demand called surplus when $t<t_{2}$. The balance point (hedging point) is at time $t^{*}=t_{1}$ and $t=t_{2}$ where the production meets the demand.

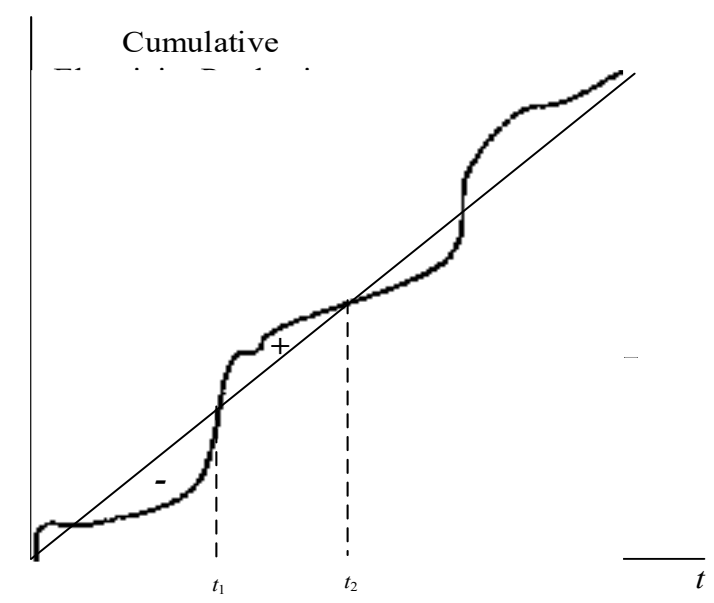

Figure 3. Energy production strategy (this behavior is modified from Gershwin's framework in [27])

\section{NUMERICAL APPROACH}

In this section, we develop the numerical method for solving the optimality conditions represented in the previous section. This method is based on Kushner's approach [25]. By adopting the algorithm in [28], the HJB equation (15) which includes the gradient of value function of $V^{i}(t, X)$ can be solved. Let $\Delta X>0$ and $\Delta t>0$ denote the length of the finite difference interval of the variable $X$ and $t$ respectively. The first-orders 
partial derivative of the value functions $V_{t}^{i}($.$) and V_{X}^{i}($.$) in equation (15) are replaced by the expressions:$

$$
\begin{aligned}
V_{t}^{i}(t, X) & =\frac{V^{i}(t+\mathrm{D} t, X)-V^{i}(t, X)}{\mathrm{D} t} \\
V_{X}^{i}(t, X) & =\frac{\frac{j}{1}_{\frac{1}{i}(t, X+\mathrm{D} X)-V^{i}(t, X)}}{\mathrm{D} X} \text { if } f(t, X, U)^{3} \quad 0 \\
& \frac{V^{i}(t, X)-V^{i}(t, X-\mathrm{D} X)}{\mathrm{D} X} \text { otherwise }
\end{aligned}
$$

Using $\Delta X$ and $\Delta t$, and after manipulations, the HJB equations can rewritten as follows:

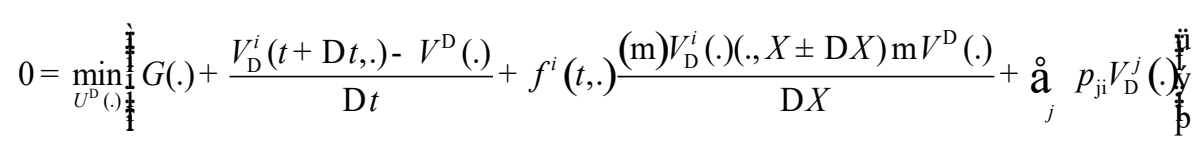

The next theorem shows that $v_{\mathrm{D}}^{i}(t, X)$ is an approximation to $v^{i}(t, X)$ for small step size $\Delta X$.

Theorem 4.1 Let $V_{\mathrm{D}}^{i}(t, X)$ denote a solution to HJB equation (22). Assume that there are constants $C_{g}$ and $K_{g}$ such that

$$
0 £ V_{\mathrm{D}}^{i}(t, X) £ C_{g}\left(1+|X|^{K_{g}}\right)
$$

then

$$
\lim _{D \circledast 0} V_{\mathrm{D}}^{i}(t, X)=V^{i}(t, X)
$$

Proof. The proof of this theorem is adopted from the one in [29] for the case of deterministic control problem. In this study, we make use of the policy improvement technique to obtain a solution of the approximating optimization problem. Let $G_{X}^{\mathrm{D}}$ and $G_{U}^{\mathrm{D}}$ be the grids of the states and control vectors belonging to the control space, the algorithm is represented as follows:

Step 1 (Initialization): Choose $e \hat{\mathrm{I}} \hat{\mathrm{A}}^{+}$. Let $k=1$ and $V_{\mathrm{D}}^{k}(t, X)=0,(t, X) \hat{\mathrm{I}}\left[t_{0}, T\right] G_{X}^{\mathrm{D}}$ and $U^{k} \hat{I} G_{U}^{\mathrm{D}}$ (initial policy).

Step 2: For a given $U^{k} \hat{\mathrm{I}} G_{U}^{\mathrm{D}}$, compute

$$
V_{\mathrm{D}}^{k-1}(t, X)=V_{\mathrm{D}}^{k}(t, X), \underline{\mathrm{U}}(t, X) \hat{\mathrm{I}}\left[t_{0}, T\right] G_{X}^{\mathrm{D}}
$$

Step 3: Compute the corresponding value function to obtain the control policy $U(t, X(t))$

Step 4: Convergence test

$$
d_{\min }=\min \left|V_{\mathrm{D}}^{k}(t, X(t))-V_{\mathrm{D}}^{k-1}(t, X(t))\right| ; d_{\max }=\max \left|V_{\mathrm{D}}^{k}(t, X(t))-V_{\mathrm{D}}^{k-1}(t, X(t))\right|
$$

If $\left|d_{\max }-d_{\min }\right| £ e$, then stop, else $k=k+1$ and go to the step 2 . 


\section{NUMERICAL EXAMPLE}

The proposed model in Section 3 is for application of hybrid wind/photovoltaic/diesel engine generator generation system such as follows: PV.

Figure 4 presents the power flow of microgrid including DG1 as DEG, DG2 as WTG, and DG3 as

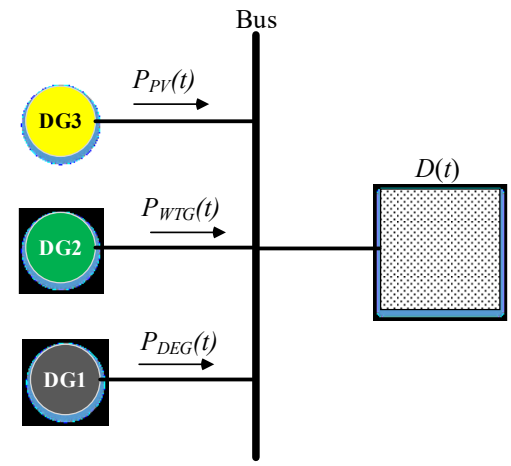

Figure 4. The power flow of MG

In this example, the forecast of load demand, PV power, and wind power generations are represented in Figure 5 to Figure 7. The constraints of system parameters in equations (4-7) for MG presents in the Table 1, these parameters also obey the Assumptions A.1 and A.2. In the Table 1, the failure rate is $\lambda=0.0001$ (i.e., the Mean Time to Failure is 10000 time units) and the repair rate is $\mu=0.09$ (i.e., the Mean Time to repair is 11.11 time units) that means the probability that the DEG fails is about $1.1 \%$, i.e., for 8760 hours in operation the DEG could be repaired in 97.3 hours (see in [27] for more details).

Table 1. Parameters of Optimal Power flow Model

\begin{tabular}{clcc}
\hline No & \multicolumn{1}{c}{ Description } & Lower & Upper \\
\hline 1 & Time interval $[\mathrm{h}]$ & 0 & 24 \\
2 & WTG power $[\mathrm{kW}]$ & 0 & 500 \\
3 & PV power $[\mathrm{kW}]$ & 0 & 600 \\
4 & DEG power $[\mathrm{kW}]$ & 1000 & 3500 \\
5 & Load demand $[\mathrm{kW}]$ & 0 & 3500 \\
6 & $C^{-}$Backlog cost $[\$ / \mathrm{kWh}]$ & 10 & - \\
7 & $C^{+}$Surplus cost $[\$ / \mathrm{kWh}]$ & 1 & - \\
8 & Failure rate $\lambda$ & 0.0001 & - \\
9 & Repair rate $\mu$ & 0.09 & - \\
\hline
\end{tabular}

The results are illustrated in Figure 8 and Figure 9. Figure 8 represents the optimal production of DEG output power versus time $t$ in the interval $[0,24 \mathrm{~h}]$. Figure 9 represents the cumulative electricity production of MG and the load demand versus time $t$. This figure shows that, the effectiveness of optimal control gives the birth to optimal power flow of MG that satisfies the load demand, thus the characteristic is linear instead of being non-linear as in Figure 3. Meanwhile, Figure 10 presents the value function which is convex function.

As the results, the novel model to hybrid wind-diesel-photovoltaic system is valid to find out the optimal power output of DEG. 


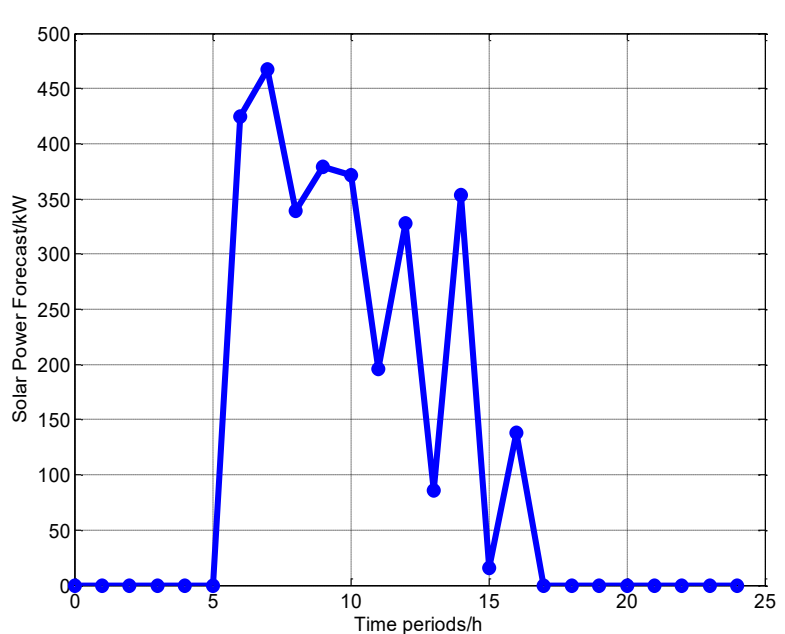

Figure 5. PV power forecast $[\mathrm{kW}]$

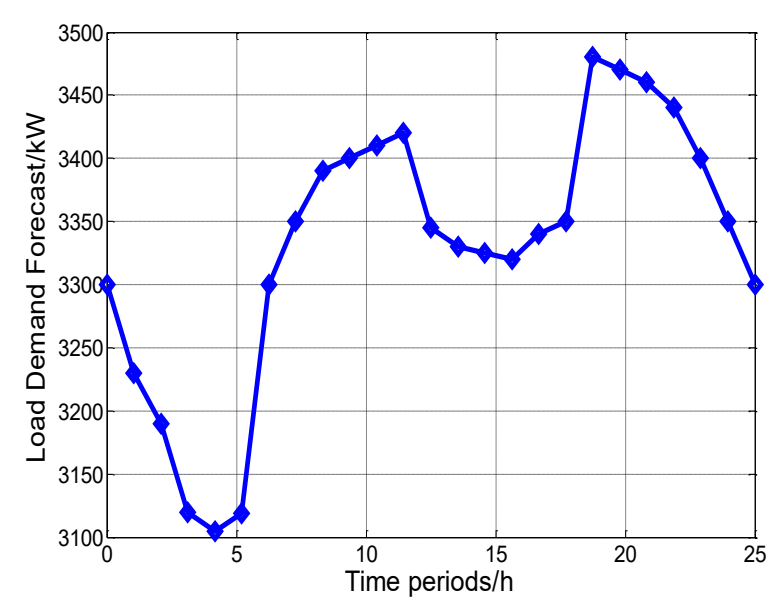

Figure 7. Demand forecast [kW]

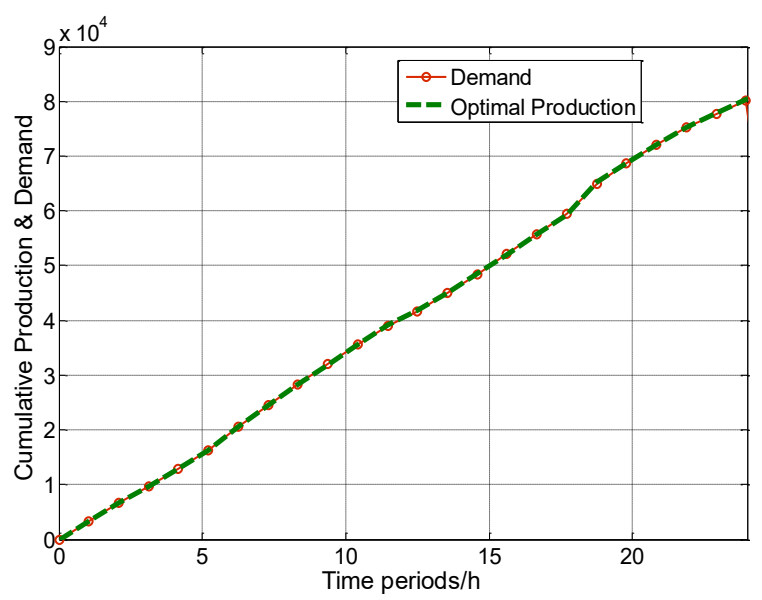

Figure 9. Cumulative production $[\mathrm{kWh}]$

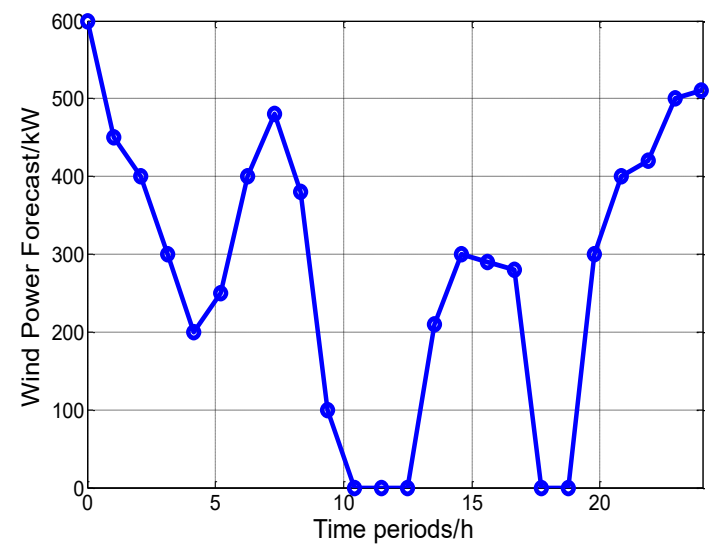

Figure 6. Wind power forecast $[\mathrm{kW}]$

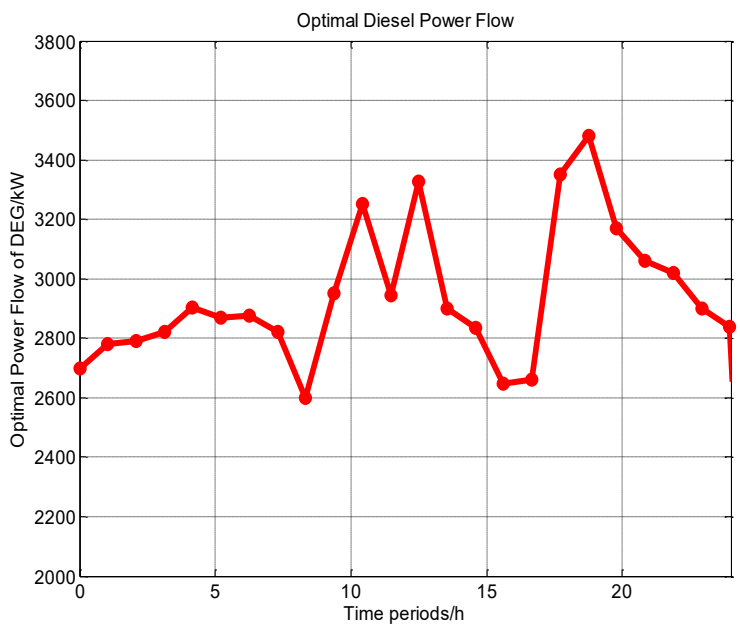

Figure 8. Optimal output power of DEG [kW]

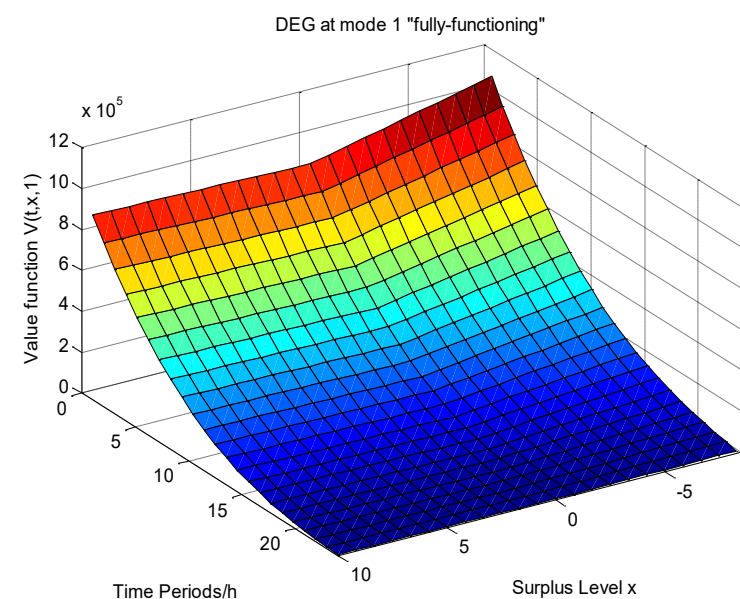

Figure 10. Value Function 


\section{SENSITIVITY ANALYSIS}

As mentioned above, the conventional implementation of state $D E G$ in this study is based on the selection of successor states and the execution of related actions. Since it is natural to use state machines to model these applications, the systemic optimal models built focused on a variety of production organization scenarios, production interactions and DEG states, as well as on stochastic market demand dynamics, WTG power, and PV power.

The new proposed model was built with a view to stochastic generation sources in parallel generating electricity, and was applied to single-DEG, WTG, and PV of islanded microgrid. The effects of the cost variable on the model are illustrated in Table 2. Consider the HPS in islanded mode, and with a fixed demand $(D=3000 \mathrm{~kW})$, WTG power $\left(P_{W T G}=1200 \mathrm{~kW}\right)$, and PV power $\left(P_{P V}=500 \mathrm{~kW}\right)$.

Table 2 Sensitivity Analysis for Varying $c^{-}$and $c^{+}$

\begin{tabular}{ccccc}
\hline Case & $c^{+}$ & $c^{-}$ & $z^{*}$ & $V\left(t, z^{*}\right)$ million \\
\hline 1 & 100 & 1000 & 0 & 88,04 \\
2 & 100 & 2000 & 0 & 162,5 \\
3 & 100 & 3000 & 0 & 237,0 \\
4 & 200 & 1000 & 0 & 88,04 \\
5 & 400 & 1000 & 0 & 88,04 \\
6 & 600 & 1000 & 0 & 88,04 \\
\hline
\end{tabular}

From a practical and a mathematical point of view, the use of the HJB equation in order to determine the optimal process cost offers a comprehensive optimization method, which renders the efficient performance of parallel machines and interactive applications feasible. The general stationary probability distribution functions for the operation mode and the repair mode, respectively, were formally investigated by determining whether the machine had sufficient average capacity to meet demands. The results obtained after the simulation of various inventory costs as shown in Table 1 and Table 2 can be interpreted as shown below.

Table 2 presents the surplus and backlog costs, the hedging point $z^{*}$, and the optimal costs at time $t=11.48 \mathrm{~h}$ which is arbitrarily chosen from within the time interval $(0,24)$. The surplus and backlog costs $\left(C^{+}, C^{-}\right)$vary between 100 and 600 units and 1000 and 3000 units, respectively. Case 1-3 in Table 2 shows that an increase in the backlog cost from 1000 to 3000 leads to the value function $V^{1}\left(t, z^{*}\right)$ increasing from 88,04 to $237,0 \mathrm{M}$. Meanwhile, the hedging point $z^{*}(\underline{t})$ does not change in order to stabilize the energy stock. For case 4-6, when the inventory costs $\mathrm{c}^{+}$are higher than 100 , the value functions $v^{1}($.$) does not change.$

The Markov model proves to be particularly suitable for modeling hybrid power systems, and its optimal control, which is based on Bellman principle, can effectively be framed with a varying demand policy that aims to optimize a given objective function. We can also see that the performance of the optimization method is stable for different manufacturing system structures. Although some assumptions have been made with regard to the processing time, the accuracy of the method is quite acceptable for general applications.

\section{CONCLUSIONS}

We have provided an analysis of an optimal power flow problem for hybrid power generation system, which underlies the well-known Markov properties. The discrete DEG states are characterized by Markov processes, and so DEG is subject to random failures and repairs, with exponential distributions of functioning and repair times. Using Markov processes in continuous time, simultaneously with Rishel's framework and a dynamic programming approach, a new model of the stochastic control problem in a deterministic horizon is formulated without a discounted rate. While the dynamic programming approach is used to make decisions in stages over time, Rishel assumptions are used to model the discrete DEG states.

In our model, we used the definitions of Markov processes found in Rishel [20] to characterize the discrete events of the DEG such a breakdown and DEG repairs. The main objective of this new model is to find the DEG power generation with minimizing the surplus cost of energy. Hence, the optimality conditions have been established and showed that the optimal generation control is the bang-bang control. On the other hand, the new model is to deal with optimal power flow control with uncertain power injection.

We applied our proposed model to real word hybrid power system: single DEG with DEG having exponential distributions of failure and repair times. The HJB equation was involved by using numerical approach. To valid our proposed model, a sensitivity evaluation has been implemented on a sample path. 


\section{REFERENCES}

[1] D. Thiam, "Renewable decentralized in developing countries: Appraisal from microgrids project in Senegal," Renew. Energy, vol. 35, no. 8, pp. 1615-1623, 2015.

[2] T. N. A. Kavousifard, "Impact of thermal recovery and hydrogen production of fuel cell power plants on distribution feeder reconfiguration," IET Gener. Transm. Distrib., vol. 6, no. November 2011, pp. 831-43, 2012.

[3] T. Tanabe et al., "Optimized operation and stabilization of microgrids with multiple energy resources," 7 th Internatonal Conf. Power Electron. ICPE'07, no. September 2007, pp. 74-78, 2008.

[4] J. M. Guerrero, J. C. Vasquez, J. Matas, L. G. De Vicuña, and M. Castilla, "Hierarchical control of droopcontrolled AC and DC microgrids - A general approach toward standardization," IEEE Trans. Ind. Electron., vol. 58, no. 1, pp. 158-172, 2011.

[5] J. C. Vasquez, J. M. Guerrero, J. Miret, M. Castilla, and L. G. De Vicuña, "Hierarchical Control of Intelligent Microgrids," IEEE Ind. Electron. Mag., no. December 2010, pp. 23-29, 2010.

[6] X. Tang, X. Hu, N. Li, W. Deng, and G. Zhang, "A novel frequency and voltage control method for islanded microgrid based on multienergy storages," IEEE Trans. Smart Grid, vol. 7, no. 1, pp. 410-419, 2016.

[7] M. Reza Ebrahimi, M. Naser Hashemnia, M. Ehsan, and A. Abbaszadeh, "A Novel Approach to Control the Frequency and Voltage of Microgrids in Islanding Operation,” Int. J. Eng. Technol., vol. 4, no. 5, pp. 562-566, 2012.

[8] Y. T. Tan, "Impact on the power system with a large penetration of photovaltaic generation," The University of Machester Institute of Science and Technology, 2004.

[9] F. Vallee, J. Lobry, and O. Deblecker, "System Reliability Assessment Method for Wind Power Integration," Power Syst. IEEE Trans., vol. 23, no. 3, pp. 1288-1297, 2008.

[10] W. El-Khattam, Y. G. Hegazy, and M. M. a. Salama, "Stochastic power flow analysis of electrical distributed generation systems," 2003 IEEE Power Eng. Soc. Gen. Meet. (IEEE Cat. No.03CH37491), vol. 2, pp. 1141-1144, 2003.

[11] C. Wang, M. Liu, and L. Guo, "Cooperative Operation and Optimal Design for Islanded Microgrid," IEEE, pp. 1-8, 2011.

[12] M. Lennard, A. Date, and X. Yu, "Islanded microgrid energy system parameter estimation using stochastic methods," Sol. Energy, vol. 147, pp. 300-313, 2017.

[13] T. Ding, Power System Operation with Large Scale Wind Power Integration. Springer, 2017.

[14] F. Laouafi, A. Boukadoum, and S. Leulmi, "A Hybrid Formulation between Differential Evolution and Simulated Annealing Algorithms for Optimal Reactive Power Dispatch," TELKOMNIKA (Telecommunication, Computing, Electronics and Control), vol. 16, no. 2, pp. 513-524, 2018.

[15] J. Zhu and W. Pan, "Intelligent Control of Wind/Photovoltaic Microgrid Information Fusion.," TELKOMNIKA (Telecommunication, Computing, Electronics and Control), vol. 15, no. 1, pp. 264-272, 2017.

[16] S. M. Antony and G. Immanuel, "A novel single phase bridgeless AC/DC PFC converter for low total harmonics distortion and high power factor," Int. J. Power Electron. Drive Syst., vol. 9, no. 1, pp. 17-24, 2018.

[17] M. K. Kim, D. H. Kim, Y. T. Yoon, S. S. Lee, and J. K. Park, "Determination of available transfer capability using continuation power flow with fuzzy set theory," 2007 IEEE Power Eng. Soc. Gen. Meet. PES, pp. 1-7, 2007.

[18] D. Villanueva, J. L. Pazos, and A. Feijóo, "Probabilistic load flow including wind power generation," IEEE Trans. Power Syst., vol. 26, no. 3, pp. 1659-1667, 2011.

[19] P. Zhang and S. T. Lee, "Probabilistic Load Flow Computation Using the Method of Combined Cumulants and Gram-Charlier Expansion,” IEEE Trans. Power Syst., vol. 19, no. 1, pp. 676-682, 2004.

[20] R. Rishel, "Control of Systems with Jump Markov Disturbances," IEEE Trans. Automat. Contr., vol. 20, no. 2, pp. 241-244, 1975.

[21] S. M. Ross, Introduction to Probability Models, 11th ed. Academic Press, 2014.

[22] R. Rishel, "Unormalized conditional probabilities and optimality for partially observed controlled jump Markov processes," Lect. notes Control Inf. Sci., pp. 326-343, 1982.

[23] E.-K. Boukas, "Commande optimale stochastique appliquée aux systèmes de production," École Polytechnique de Montréal, 1987.

[24] W. H. Fleming and H. M. Soner, Controlled Markov Processes and Viscosity Solutions, Second. Springer.

[25] H. J. Kushner and Paul Dupuis, Numerical Methods for Stochastic Control Problems in Continuous Time, Second. Springer, 2001.

[26] D. P. Bertsekas, "Dynamic Programming and Optimal Control: Volume I." Athena Scientific, Belmont, Massachusetts, p. 543, 2017.

[27] S. B. Gershwin, "Manufacturing Systems Engineering," p. 350, 1994.

[28] E. Charlot, J. P. Kenné, and S. Nadeau, "Optimal production, maintenance and lockout/tagout control policies in manufacturing systems," Int. J. Prod. Econ., vol. 107, no. 2, pp. 435-450, 2007.

[29] H. Yan and Q. Zhang, "A numerical method in Optimal production and Setup Scheduling of Stochastic Manufacturing Systems," IEEE Trans. Automat. Contr., vol. 42, no. 10, pp. 1452-1455, 1997. 


\section{BIOGRAPHIES OF AUTHORS}
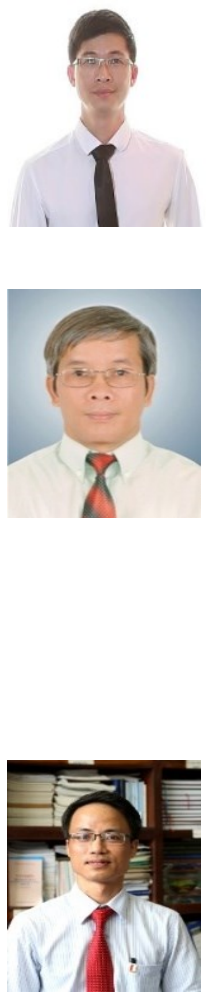

Thang Diep received the B.S degree from Department of Electric Power System, Hanoi University of Science and Technology (HUST), Hanoi, Vietnam, in 2002, the Master's degree in Electrical Engineering from Institut National Polytechnique de Grenoble, Grenoble, France, in 2004, and the Master of Engieering in Mechanical Engineering from École de Technologie Supérieure, Montreal, Québec, Canada, in 2011. He is currently with Institute for Control Engineering and Automation at HUST as Ph.D student. His Research Interests are Hybrid Power System, MG, Optimal Power Flow, Optimal Control, Stochastic Optimal Control, Smart Grid.

Quang Nguyen-Phung received his Dipl.-Ing. (Uni.), Dr.-Ing. and Dr.-Ing. habil. degrees from TU Dresden, Germany in 1975, 1991 and 1994 respectively. Prior to his return to Vietnam, he had worked in Germany industry for many years, contributed to create inverters REFU 402 Vectovar, RD500 (REFU Elektronik); Simovert 6SE42, Master Drive MC (Siemens). From 1996 to 1998, he served as lecturer of TU Dresden where he was conferred as Privatdozent in 1997. He joined Hanoi University of Science and Technology in 1999, as lecturer up to now. He is currently a professor of HUST and honorary professor of TU Dresden. He was author/co-author of more than 160 journal and conference papers; 8 books with three among them was written in German and one in English entitled "Vector Control of Three-Phase AC Machines - System Development in the Practice" published by Springer in 2008, and 2nd edition in June 2015. His Research Interests are Electrical Drive Systems, Motion Control, Robotic Control, Vector Control of Electrical Machines, Wind and Solar Power Systems, Digital Control Systems, Modeling and Simulation.

Huy Nguyen-Duc received the B.S degree from Department of Electric Power System, Hanoi University of Science and Technology (HUST), Hanoi, Vietnam, in 2001, and the Ph.D degree in Electrical Engineering from Department of Electrical Engineering, École de Technologie Supérieure, Montreal, Québec, Canada, in 2011. He is currently with the Department of Electric Power System at HUST. He is a member of the IEEE. His Research Interests are Power System Operation and Control, Application of Artificial Intelligence Technique in Power Systems. 